Technical Note

\title{
In Vitro Expansion of CAG, CAA, and Mixed CAG/CAA Repeats
}

\author{
Grzegorz Figura, Edyta Koscianska and Wlodzimierz J. Krzyzosiak * \\ Department of Molecular Biomedicine, Institute of Bioorganic Chemistry, \\ Polish Academy of Sciences, Noskowskiego 12/14 Str., 61-704 Poznan, Poland; \\ E-Mails: gfigura@man.poznan.pl (G.F.); edytak@ibch.poznan.pl (E.K.) \\ * Author to whom correspondence should be addressed; E-Mail: wlodkrzy@ibch.poznan.pl; \\ Tel.: +48-61-852-8503; Fax: +48-61-852-0532.
}

Academic Editor: Mateus Webba da Silva

Received: 27 July 2015 / Accepted: 4 August 2015 / Published: 11 August 2015

\begin{abstract}
Polyglutamine diseases, including Huntington's disease and a number of spinocerebellar ataxias, are caused by expanded CAG repeats that are located in translated sequences of individual, functionally-unrelated genes. Only mutant proteins containing polyglutamine expansions have long been thought to be pathogenic, but recent evidence has implicated mutant transcripts containing long CAG repeats in pathogenic processes. The presence of two pathogenic factors prompted us to attempt to distinguish the effects triggered by mutant protein from those caused by mutant RNA in cellular models of polyglutamine diseases. We used the SLIP (Synthesis of Long Iterative Polynucleotide) method to generate plasmids expressing long CAG repeats (forming a hairpin structure), CAA-interrupted CAG repeats (forming multiple unstable hairpins) or pure CAA repeats (not forming any secondary structure). We successfully modified the original SLIP protocol to generate repeats of desired length starting from constructs containing short repeat tracts. We demonstrated that the SLIP method is a time- and cost-effective approach to manipulate the lengths of expanded repeat sequences.
\end{abstract}

Keywords: SLIP; trinucleotide repeats; in vitro expansion; repeats in vitro cloning; polyglutamine diseases; plasmid constructs 


\section{Introduction}

At least fifteen human hereditary neurological diseases result from the expansion of unstable triplet repeats in single genes [1]. Nine of these disorders, mostly neurodegenerative, are caused by expanded CAG repeats located in translated sequences [2]. This group of disorders, known as polyglutamine (polyQ) diseases, includes Huntington's disease, spinocerebellar ataxia types 1, 2, 3, 6, 7, and 17, spinobulbar muscular atrophy, and dentatorubral-pallidoluysian atrophy. The main type of pathogenic mechanism associated with this group of disorders is a mutant protein gain-of-function [2]. However, there is growing evidence for the contribution of mutant transcript toxicity, which resembles, to the same extent, the toxicity observed in myotonic dystrophy type 1 [3-5].

One way to assess the molecular mechanisms of pathogenesis in this group of disorders is to generate and investigate cellular models of polyQ diseases expressing repeated sequences of determined length and structure [6-8]. The existing models developed for this purpose were recently reviewed [5]. The development of such models typically requires assembly of genetic constructs from which the repeats are expressed. However, manipulating the length of CAG repeats encoding a polyQ tract in a controlled way is not simple. This is due to the hairpin structure formed by the repeats [9], DNA polymerase slippage on repeated sequences [10], and their instability in cells [11].

To address these problems, several methods of cloning repeated sequences have been developed. The commonly used method involves self-priming of two oligonucleotides, one containing CAG repeats and another containing complementary CTG repeats. The method has several modifications which differ in the PCR conditions used [12-17]. Additionally, PCR-free methods have been proposed, which involve repeated cycles of plasmid digestion with restriction enzymes and ligation of another repeat module [18]. Another approach involves the insertion of mixed CAG/CAA repeats into CAG repeat tracts using SII-type restriction enzymes to increase the repeats' stability [19].

In this study, we assessed the in vitro CAG repeat expansion method known as SLIP (Synthesis of Long Iterative Polynucleotide) [15]. This method involves plasmid digestion with a restriction enzyme, a single PCR cycle, and bacterial transformation. We optimized SLIP to expand CAG, CAA and mixed $(\mathrm{CAG})_{3} \mathrm{CAA}$ repeats. Both $\mathrm{CAG}$ and $\mathrm{CAA}$ triplets encode glutamine, although the codon usage of CAG is three times higher than that of CAA in human cells [20]. In the ATXN2 and TBP transcripts implicated in spinocerebellar ataxia type 2 and type 17, most of the normal variants of these genes contain CAA-interrupted CAG repeats [21-28], which have been shown to strongly alter the structures formed by pure CAG repeats. However, we could not take advantage of these natural repeat insertion patterns because of the restrictions of the SLIP method [29].

Thus, the main reason for performing our SLIP experiments with this particular set of repeated sequence motifs is the ability of their long tracts to form different structures in transcripts. The CAG repeats form long, undisturbed hairpin structures, the CAA-interrupted CAG repeats form multiple short and less stable hairpins, and long stretches of CAA repeats remain single-stranded [30]. With cellular models expressing such variants of the glutamine-coding repeats, it should be possible to distinguish between the effects caused by structure-dependent RNA toxicity and protein toxicity in polyQ diseases [5]. The importance of such an approach can be stressed by the lack of correlation between the presence of polyQ inclusions and disease, suggesting that other mechanisms, such as RNA-based toxicity, are likely to be involved. 


\section{Results and Discussion}

\subsection{Experimental Design}

We intended to create genetic constructs expressing the human ATXN3 gene, which is responsible for spinocerebellar ataxia type 3 and contains three types of repeated sequence of a defined length of approximately 60, 90, and 120 triplet repeats. The starting constructs required by the SLIP method were prepared by using standard molecular cloning techniques. The construct bearing human cDNA for ATXN3, containing $69 \mathrm{CAG}$ repeats and flanked by BsmBI and Eco0109I sites, was cloned into pGEM $^{\circledR}$-T Easy vector (Promega, Madison, WI, USA) as described previously [31].

To generate the starting constructs of pure CAA repeats and CAA-interrupted CAG repeats, we used synthetic oligonucleotides containing 20 such repeats. Oligonucleotides were annealed and cloned into a $69 \mathrm{CAG}$ construct by digesting the original repeat tract with restriction enzymes and ligating the CAA or CAA-interrupted CAG repeat tract into the construct. This procedure swapped the original $69 \mathrm{CAG}$ repeats with either $20 \mathrm{CAA}$ or $20 \mathrm{CAA}$-interrupted CAG repeats. The resultant constructs were then subjected to repeat tract expansion by using the SLIP method. The CAG, CAA, and CAA-interrupted CAG repeat constructs were digested with either BsmBI or Eco0109I restriction enzymes, respectively, 48 bp upstream and $3 \mathrm{bp}$ downstream of the repeat tract. Pairs of single enzyme digestion products from each starting construct were then combined, subjected to one PCR cycle, and used for bacterial transformation. The colony PCR products were analyzed on agarose gels to assess repeat sequence length changes.

The SLIP method requires the presence of a repetitive sequence module to be effective. Products of independent, parallel digestions anneal to each other imperfectly, which allows the DNA polymerase to extend the repeat tract. The polymerase has to exhibit $3^{\prime}$ exonuclease activity to remove the non-annealed fragments of the specific sequence flanking the repeat tract (Figure 1).

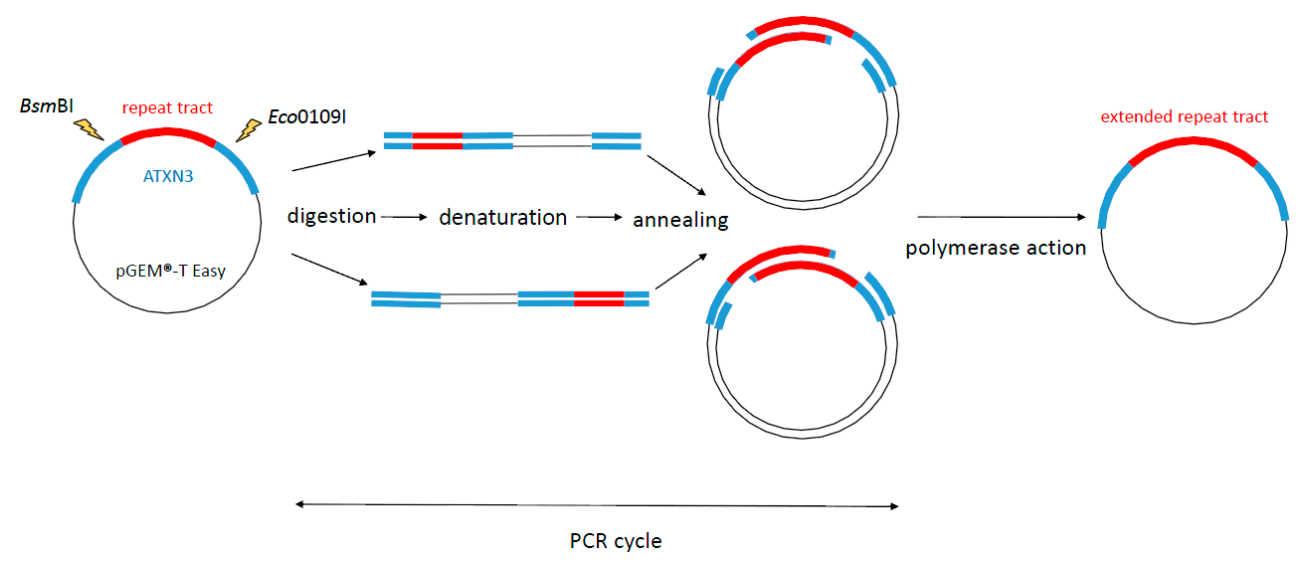

Figure 1. Extension of a repeat tract by using the SLIP method. Starting constructs are independently digested with enzymes that digest upstream and downstream sites of the repeat tract. The digestion products are then combined and subjected to a single PCR cycle. Repeat extension by gap-filling is carried out by using a polymerase that extends the products of different digestions which repeated sequences are not perfectly aligned. The polymerase first removes mismatched sequences immediately adjacent to the repeat tract and then extends the $3^{\prime}$ end, resulting in repeat tract elongation [15]. 
The pattern of CAG repeat interruption by CAA triplets was chosen from several considered variants, including $(\mathrm{CAG})_{4}(\mathrm{CAA})_{2},(\mathrm{CAG})_{3}(\mathrm{CAA})_{2},(\mathrm{CAG})_{3} \mathrm{CAA}$, and $(\mathrm{CAG})_{2} \mathrm{CAA}$, based on Mfold-predicted structures of these variants in transcripts (Figure 2). For SLIP, we selected the sequence motif (CAG) ${ }_{3} \mathrm{CAA}$ (variant $\mathrm{D}$ ) due to its low frequency of CAA triplets, having lower codon usage than $\mathrm{CAG}$, the second smallest repeat module size, and sufficient hairpin structure destabilizing ability [29].

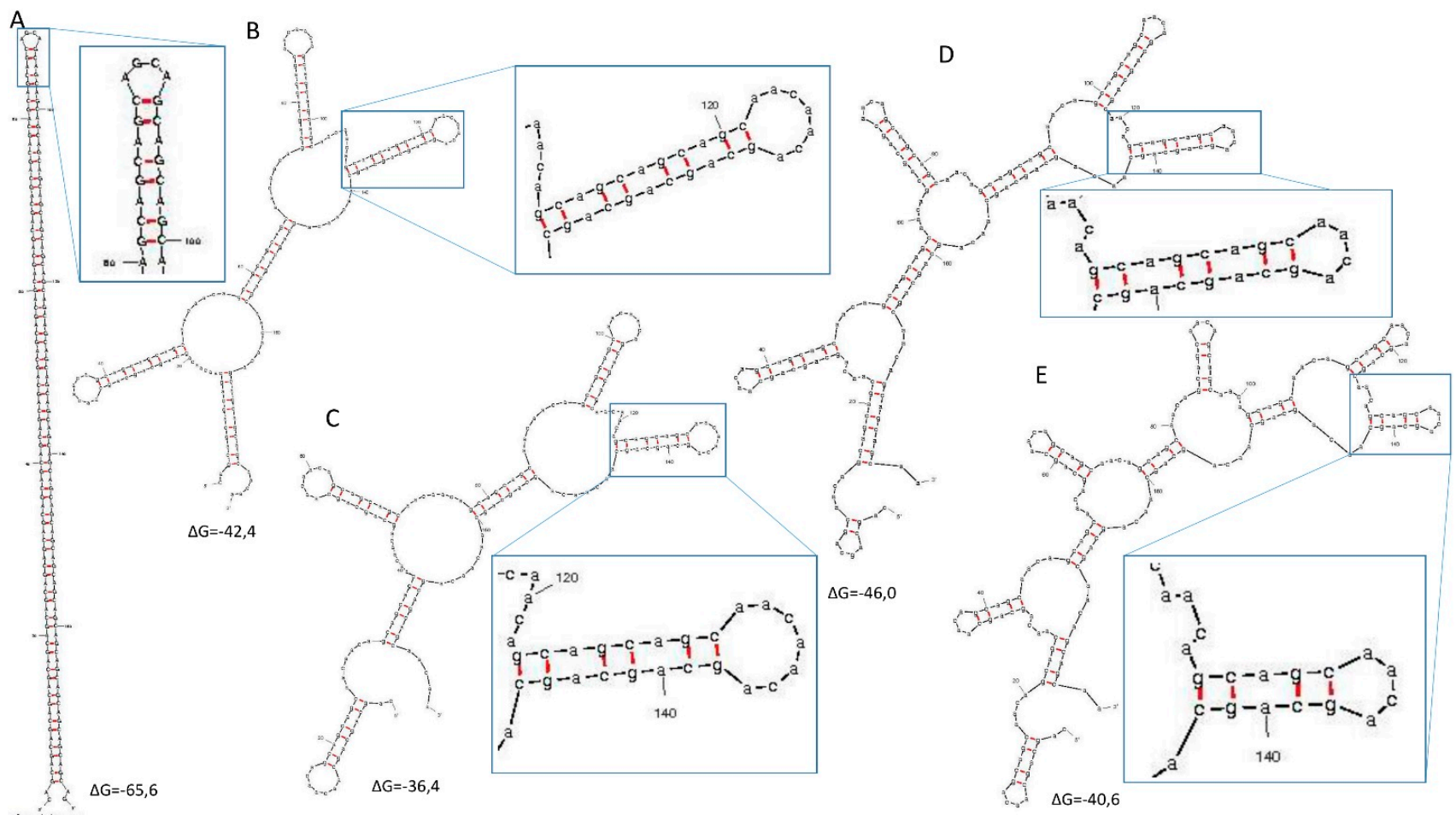

Figure 2. RNA structures of different repeat tracts containing 60 repeats predicted using Mfold [32-34]. Predictions were made using default settings, and structures having the lowest free energy of formation are presented. (A) Hairpin structure predicted for the pure CAG repeat tract; $(\mathbf{B})$ the $(\mathrm{CAG})_{4}(\mathrm{CAA})_{2}$ repeat tract; $(\mathbf{C})$ the $(\mathrm{CAG})_{3}(\mathrm{CAA})_{2}$ tract; $(\mathbf{D})$ the $(\mathrm{CAG})_{3} \mathrm{CAA}$ tract; and $(\mathbf{E})$ the $(\mathrm{CAG})_{2} \mathrm{CAA}$ tract.

\subsection{SLIP (Synthesis of Long Iterative Polynucleotide) Performance}

By performing multiple rounds of SLIP, it was possible to generate numerous variants of starting constructs that differed in repeat sequence length. Each SLIP round takes no more than three days. Day 1 involves construct digestion with restriction enzymes, PCR and bacterial transformation. Day 2 involves screening of clones by colony PCR and setting up bacterial cultures for DNA preparation. Day 3 involves DNA preparation; at this point, the system is ready for the next round of SLIP. The extent of the repeat length change is difficult to control or predict. SLIP causes not only repeat tract expansion but also contraction. When repeat sequence changes occur at a low frequency, it is necessary to screen more clones (sometimes more than 100) to find one that is expanded or contracted. Representative gels from SLIP experiments with our three types of repeats are presented in Figure 3. Panels A and B show the raw SLIP data for the CAG repeat, panels C and D show such data for the $\mathrm{CAA}$ repeat, and panels $\mathrm{E}$ and $\mathrm{F}$ for the $(\mathrm{CAG}){ }_{3} \mathrm{CAA}$ repeat. 

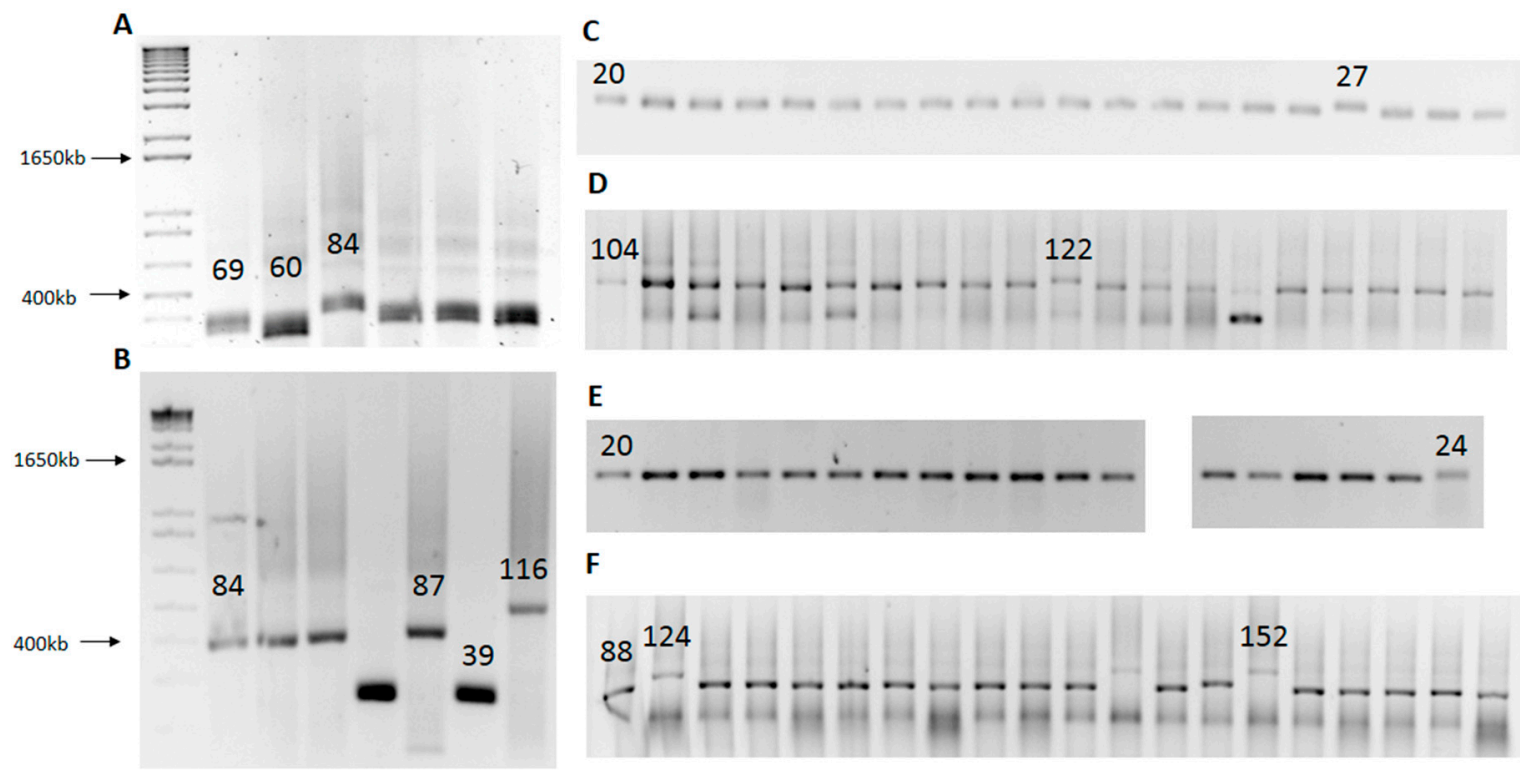

Figure 3. Screen of $A T X N 3$ constructs generated by using the SLIP method. SLIP of ATXN3 with, (A) 69 CAG repeats; (B) 84 CAG repeats; (C) 20 CAA repeats; (D) 104 CAA repeats; (E) 20 CAA-interrupted CAG (1:3 ratio) repeats; and (F) 88 CAA-interrupted CAG (1:3 ratio) repeats. The numbers above the bands indicate the length of the repeat tract confirmed by sequencing. The first PCR product in the gel represents a control PCR product from a construct subjected to SLIP.

Table 1 represents the outcomes of consecutive rounds of SLIP experiments performed with three types of repeats.

Table 1. Results of a series of SLIP experiments showing the efficiency of elongation of a particular repeat tract. L, the number of elongated clones; $\mathrm{S}$, the number of shortened clones; total, the number of screened clones; "-" indicates that SLIP was not performed. When several variants of repeat length were obtained and confirmed by sequencing, the longest one was used in additional experiments. For 104 CAA and 36 CAG/CAA variants, two SLIP experiments were performed.

\begin{tabular}{|c|c|c|c|c|c|c|c|c|c|c|c|}
\hline CAG & $\mathbf{L}$ & S & Total & $\mathrm{CAG} / \mathrm{CAA}$ & $\mathbf{L}$ & $\mathbf{S}$ & Total & CAA & $\mathbf{L}$ & $\mathbf{S}$ & Total \\
\hline 69 & 3 & 3 & 25 & 20 & 1 & 0 & 85 & 20 & 2 & 0 & 95 \\
\hline 84 & 2 & 2 & 18 & 24 & 1 & 1 & 95 & 25,27 & 2 & 1 & 228 \\
\hline \multirow[t]{9}{*}{87,116} & - & - & - & 28 & 2 & 0 & 114 & 30,33 & 1 & 0 & 114 \\
\hline & & & & 36 & 4 & 1 & 114 & 36 & 1 & 0 & 114 \\
\hline & & & & 30 & 7 & 2 & 114 & 30 & 1 & & \\
\hline & & & & $40,44,48,52$ & 2 & 3 & 114 & 53 & 2 & 2 & 228 \\
\hline & & & & 80,88 & 7 & 2 & 114 & 58 & 1 & 2 & 114 \\
\hline & & & & $96,108,116$ & - & - & - & 104 & 6 & 3 & 114 \\
\hline & & & & 124,152 & - & - & & & 1 & 5 & 114 \\
\hline & & & & & & & & 143,150 & - & - & - \\
\hline & & & & & & & & 122 & & & \\
\hline
\end{tabular}


As shown in this table, the expansion size is random, and short repeats are less prone to expand than long repeats, which is consistent with the nature of the SLIP method (Figure 1). Short tracts offer fewer possibilities for alternative annealing during the PCR cycle and, thus, their elongation is less likely. All CAG repeat variants were obtained using the original SLIP method [15].

We found that expansion of $20 \mathrm{CAA}$ and $20 \mathrm{CAA}$-interrupted CAG repeats did not occur under conditions used to successfully elongate the $69 \mathrm{CAG}$ starting construct (annealing temperature $55^{\circ} \mathrm{C}$ ). Therefore, we performed SLIP with two short starting constructs at lower annealing temperatures of 50,45 , and $40{ }^{\circ} \mathrm{C}$. Only at the two latter conditions were expansion products detected. Two expanded products containing 27 and 25 repeats were obtained at $45^{\circ} \mathrm{C}$ from the 20 CAA construct and one with 28 repeats from the $20 \mathrm{CAA}$-interrupted CAG construct. The results of SLIP were also compared for the $104 \mathrm{CAA}$ repeat tract annealed at two different temperatures. At $40{ }^{\circ} \mathrm{C}$, six elongated repeats and three contracted repeats were formed. The two longest expansions contained 143 and $150 \mathrm{CAA}$ repeats. At $45{ }^{\circ} \mathrm{C}$, one elongated clone containing a $122 \mathrm{CAA}$ repeat tract, and five contracted clones were generated.

Different types of repeats, i.e., CAG, CAA, and (CAG) $)_{3} \mathrm{CAA}$, appear to have different abilities to change their length during SLIP, which can be noted by comparing the number of expanded and contracted clones with the total number of screened clones. For example, from a 69 CAG starting tract, SLIP generated six clones with changed repeat length out of 25 clones screened. For a 58 CAA starting tract, three such clones were detected out of 114 screened, and from a 52 CAA-interrupted CAG starting tract, five out of 114 (Table 1). The tracts most susceptible to variations in length were the $\mathrm{CAG}$ repeat, followed by the $(\mathrm{CAG})_{3} \mathrm{CAA}$ repeat and then by the pure CAA repeat. This finding may reflect that different structures formed by these three types of DNA repeats under the conditions of the experiment. Structure prediction shows that the $\mathrm{CAG}$ repeat tract forms a long hairpin structure (Figure 4A), (CAG) ${ }_{3} \mathrm{CAA}$ forms numerous shorter hairpins separated by single-stranded fragments (Figure 4B), and the CAA repeat, as in RNA, does not form any secondary structure. Hairpin structure formation probably facilitates imperfect alignments of repeated sequences, providing more room for expansion and contraction events. Figure 4C presents selected hypothetical structures of annealed restriction enzyme digestion products formed by three types of repeats used for SLIP. These models explain the varying expansion capabilities of these repeats. 


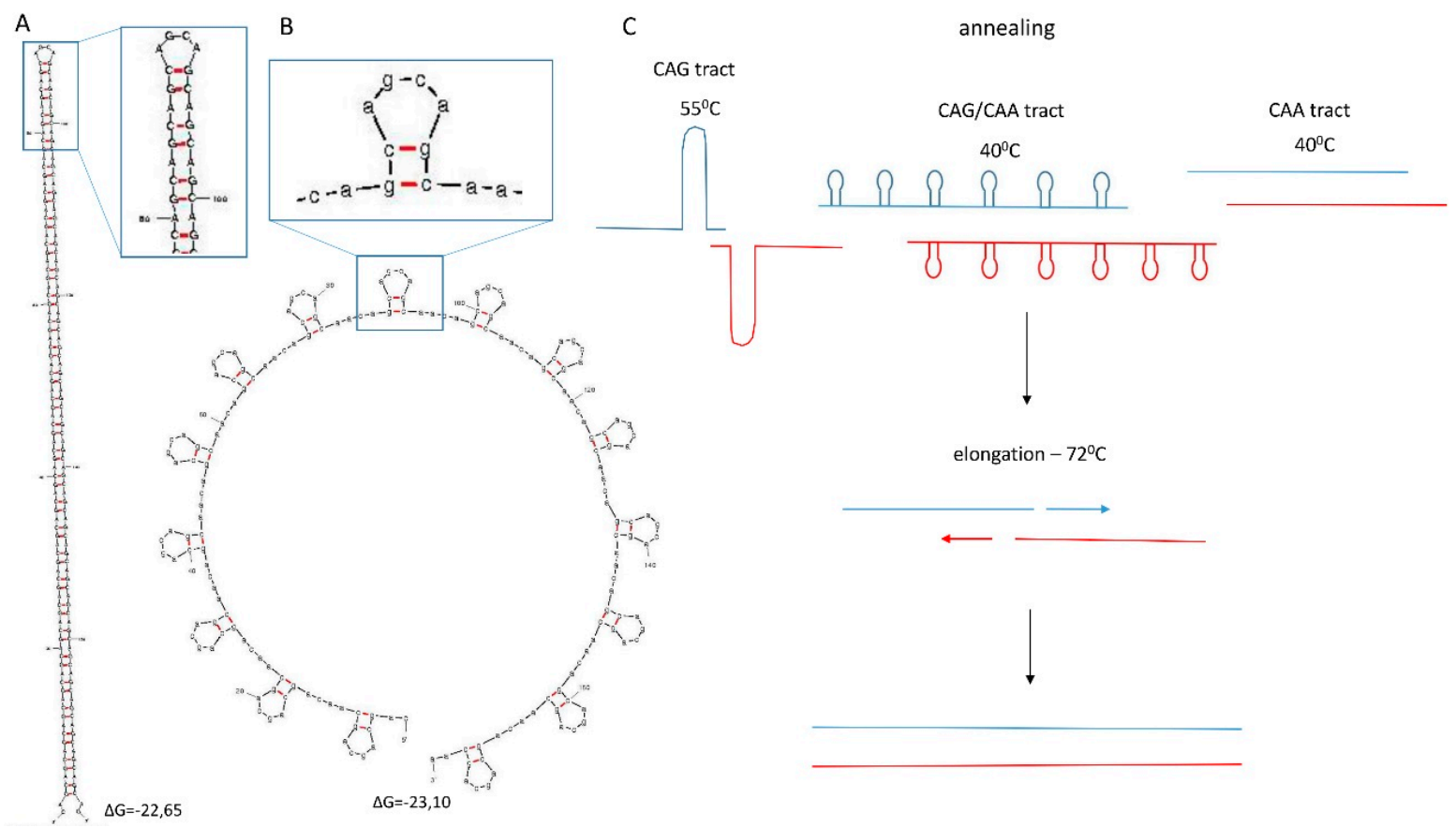

Figure 4. Structure of $\mathrm{CAG}$ and $(\mathrm{CAG})_{3} \mathrm{CAA}$ repeat tracts predicted for 60 triplets using Mfold (DNA folding form), and hypothetical hairpin structure behavior during SLIP. Red and blue colors indicate products of BsmBI and Eco0109I restriction enzymes digestions. Predictions were performed at default settings using an annealing temperature corresponding to the temperature used during the PCR cycle. (A) CAG repeat structure at $55{ }^{\circ} \mathrm{C}$; (B) (CAG) $3 \mathrm{CAA}$ repeat structure at $40{ }^{\circ} \mathrm{C}$. Only the lowest energy structures are presented; (C) hypothetical behavior of repeat tracts during SLIP. At annealing temperature, hairpin structures are relatively stable, with $\Delta \mathrm{G}=-22.65 \mathrm{kcal} / \mathrm{mol}$ for the CAG tract and $\Delta \mathrm{G}=-23.15 \mathrm{kcal} / \mathrm{mol}$ for the CAG/CAA tract; at $72{ }^{\circ} \mathrm{C}$, the $\Delta \mathrm{G}$ rises to -6.1 and 0.62 , respectively, making hairpins less stable and allowing polymerase to function [32-34].

\section{Experimental Section}

\subsection{SLIP (Synthesis of Long Iterative Polynucleotide)}

SLIP was performed as described previously by Takahashi et al. [15], with modifications described in the main text. Briefly, DNA constructs were digested in parallel using Eco0109I FastDigest (Thermo Scientific, Waltham, MA, USA) and BsmBI FastDigest (Thermo Scientific, Waltham, MA, USA) enzymes for $40 \mathrm{~min}$ at $37{ }^{\circ} \mathrm{C}$, followed by $10 \mathrm{~min}$ at $65{ }^{\circ} \mathrm{C}$ for enzyme inactivation. Digestion products were then combined and subjected to one PCR cycle followed by DH5 $\alpha$ bacterial transformation.

\subsection{Colony PCR}

Colony PCR was performed using the forward primer 5'GGAAGAGACGAGAAGCCTAC and the reverse primer 5'TCACCTAGATCACTCCCAAGT. The PCR cycling conditions were an initial denaturation at $95{ }^{\circ} \mathrm{C}$ for $5 \mathrm{~min}$, denaturation at $95{ }^{\circ} \mathrm{C}$ for $30 \mathrm{~s}$, annealing at $60{ }^{\circ} \mathrm{C}$ for $15 \mathrm{~s}$, extension 
at $72{ }^{\circ} \mathrm{C}$ for $30 \mathrm{~s} \times 30$ cycles and a final elongation at $72{ }^{\circ} \mathrm{C}$ for $7 \mathrm{~min}$. The reaction mixture in the final volume of $5 \mu \mathrm{L}$ contained $0.5 \mu \mathrm{M}$ forward primer, $0.5 \mu \mathrm{M}$ reverse primer, $1 \mathrm{mM} \mathrm{dNTP}$ mix, $1.25 \mathrm{mM} \mathrm{Mg}, 1 \times$ GoTaq Flexi buffer and 0.0625 units GoTaq G2 Flexi polymerase (Promega, Madison, WI, USA). PCR products were than analyzed on an $1 \%$ agarose gel.

\subsection{Preparation of Starting Constructs}

Synthetic oligonucleotides with Eco0109I and BsmBI ends were synthesized (IBB Warsaw) (Table 2). The oligomers were annealed by heating and gradual cooling. The oligomers were subsequently cloned into the $69 \mathrm{CAG}$ starting constructs that were previously digested with Eco0109I FastDigest (Thermo Scientific, Waltham, MA, USA) and BsmBI FastDigest (Thermo Scientific, Waltham, MA, USA) enzymes and purified by excising agarose gel (TAE buffer: $40 \mathrm{mM}$ Trizma base (Sigma-Aldrich, St. Louis, MO, USA), $20 \mathrm{mM}$ glacial acetic acid (Avantor Performance Materials Poland S.A., Gliwice, Poland), 1 mM EDTA (Avantor Performance Materials Poland S.A., Gliwice, Poland) using the Gel-Out Kit (A\&A Biotechnology, Gdynia, Poland). Ligation was performed overnight at $4{ }^{\circ} \mathrm{C}$ with $50 \mathrm{ng}$ of vector and a $6: 1$ insert/vector molar ratio using T4 DNA ligase (Promega, Madison, WI, USA). The next day, the entire reaction mixture was used to transform DH5 $\alpha$ bacteria, and the obtained colonies were screened by colony PCR as described above. Plasmids from colonies containing constructs with an appropriate length of repeat tract were miniprepped using the Plasmid Mini Kit (A\&A Biotechnology, Gdynia, Poland). The nucleotide sequence of the obtained constructs was confirmed by DNA sequencing.

Table 2. Sequences of synthetic oligonucleotides. Repeat tracts are shown in red and restriction sites are underlined.

\begin{tabular}{|c|c|}
\hline Repeated Motif & Oligonucleotide Sequence \\
\hline$(\mathrm{CAG})_{3} \mathrm{CAA}$ & $\begin{array}{r}5 \text { 'cggaagagacgagaagcctactttgaaaaacagcagcaaaagcagcaacagcagcagcaacagcagcagcaacagca } \\
\text { gcagcaacagcagcagcaacagcagcagcaacgg }(111 \mathrm{nt})\end{array}$ \\
\hline$(\mathrm{CAG})_{3} \mathrm{CAA}$ & $\begin{array}{c}5^{\prime} \text { gtccegttgctgetgetgttgctgetgetgttgctgetgetgttgctgetgetgttgctgetgetgttgctgettttgctgetgttttc } \\
\text { aaagtaggcttctcgtctct }(107 \mathrm{nt})\end{array}$ \\
\hline CAA & $\begin{array}{r}5 \text { 'cggaagagacgagaagcctactttgaaaaacagcagcaaaagcagcaacaacaacaacaacaacaacaacaacaacaa } \\
\text { caacaacaacaacaacaacaacaacaacaacgg }(111 \mathrm{nt})\end{array}$ \\
\hline CAA & $\begin{array}{c}\begin{array}{l}5 \text { 'gtcccgttgttgttgttgttgttgttgttgttgttgttgttgttgttgttgttgttgttgttgttgttgetgettttgctgetgttttcaaagt } \\
\text { aggcttctcgtctct }(107 \mathrm{nt})\end{array}\end{array}$ \\
\hline
\end{tabular}

\section{Conclusions}

The SLIP method is fast and easy to perform; the entire cycle of repeat length variant synthesis and analysis can be completed within three days. We found this method to be very effective in generating $\sim 60, \sim 90$, and $\sim 120$ repeat length variants of CAG, CAA, and (CAG) ${ }_{3} \mathrm{CAA}$ repeat tracts. The latter two repeats were expanded by SLIP for the first time, starting from constructs containing very short repeat tracts of only 20 repeats. In these cases, the repeat length changes occurred less frequently, which required screening higher numbers of clones. For the SLIP method, the repeat tract needs to be flanked by nearby restriction enzyme cleavage sites. In our system, the restriction enzyme sites were located $48 \mathrm{bp}$ upstream and $3 \mathrm{bp}$ downstream of the repeat tract; therefore, the SLIP method can tolerate this 
distance. The exact outcome of the SLIP application, in terms of the size of the expansion or contraction, is difficult to control. In our study, the annealing temperature used in the PCR step was the major factor affecting the SLIP results.

The SLIP method does not need sub-cloning steps and may be used to manipulate the length of the repeat tract directly inside the gene construct. Moreover, SLIP has a higher repeat generation limit compared to PCR-based methods [15]; however, it requires starting constructs which may be considered disadvantageous. In comparison to PCR-free methods, SLIP enables generation of the desired repeat length tracts in fewer steps and is less laborious.

In conclusion, our results demonstrate that SLIP is indeed a versatile method for extension and/or contraction of repeated sequences, and that this method is not restricted to manipulation of long repeats forming stable secondary structures. The ATXN3 constructs, described in this study, have been sub-cloned into expression vectors and transfected human neuroblastoma SK-N-MC cells have been analyzed for expression of RNA toxicity markers (manuscript in preparation).

\section{Acknowledgments}

This work was supported by funding from the National Science Center (2012/06/A/NZ1/00094 to Wlodzimierz J. Krzyzosiak). This publication was also supported by the Polish Ministry of Science and Higher Education, under the Leading National Research Centre (KNOW) program for the years 2014-2019.

\section{Author Contributions}

Wlodzimierz J. Krzyzosiak, Edyta Koscianska and Grzegorz Figura conceived and designed the study; Grzegorz Figura performed the experiments; Edyta Koscianska, Grzegorz Figura and Wlodzimierz J. Krzyzosiak analazyd data; Edyta Koscianska, Grzegorz Figura and Wlodzimierz J. Krzyzosiak wrote the paper.

\section{Conflicts of Interest}

The authors declare no conflict of interest.

\section{References}

1. López Castel, A.; Cleary, J.D.; Pearson, C.E. Repeat instability as the basis for human diseases and as a potential target for therapy. Nat. Rev. Mol. Cell Biol. 2010, 11, 165-170.

2. Ashizawa, T.; Wells, R.D. Overview of the field. In Genetic Instabilities and Neurological Diseases, 2nd ed.; Wells, R.D., Ashizawa, T., Eds.; Elsevier: Burlington, MA, USA, 2006; pp. 3-17.

3. Ranum, L.P.; Cooper, T.A. RNA-mediated neuromuscular disorders. Annu. Rev. Neurosci. 2006, 29, 259-277.

4. Wheeler, T.M.; Thornton, C.A. Myotonic dystrophy: RNA-mediated muscle disease. Curr. Opin. Neurol. 2007, 20, 572-576.

5. Fiszer, A.; Krzyzosiak, W.J. RNA toxicity in polyglutamine disorders: Concepts, models, and progress of research. J. Mol. Med. 2013, 91, 683-691. 
6. Ho, T.H.; Savkur, R.S.; Poulos, M.G.; Mancini, M.A.; Swanson, M.S.; Cooper, T.A. Colocalization of muscleblind with RNA foci is separable from mis-regulation of alternative splicing in myotonic dystrophy. J. Cell Sci. 2005, 118, 2923-2933.

7. Mykowska, A.; Sobczak, K.; Wojciechowska, M.; Kozlowski, P.; Krzyzosiak, W.J. CAG repeats mimic CUG repeats in the misregulation of alternative splicing. Nucleic Acids Res. 2011, 39, 8938-8951.

8. Bañez-Coronel, M.; Porta, S.; Kagerbauer, B.; Mateu-Huertas, E.; Pantano, L.; Ferrer, I.; Guzmán, M.; Estivill, X.; Martí, E. A pathogenic mechanism in huntington's disease involves small CAG-repeated RNAs with neurotoxic activity. PLoS Genet. 2012, 8, e1002481.

9. Usdin, K. Bending the rules: Unusual nucleic acid structures and disease pathology in the repeat expansion diseases. In Genetic Instabilities and Neurological Diseases, 2nd ed.; Wells, R.D., Ashizawa, T., Eds.; Elsevier: Burlington, MA, USA, 2006; pp. 617-635.

10. Mirkin, S.M. Replication of expandable DNA repeats. In Genetic Instabilities and Neurological Diseases, 2nd ed.; Wells, R.D., Ashizawa, T., Eds.; Elsevier: Burlington, MA, USA, 2006; pp. 637-644.

11. Lin, Y.; Dion, V.; Wilson, J.H. Transcription and triplet repeat instability. In Genetic Instabilities and Neurological Diseases, 2nd ed.; Wells, R.D., Ashizawa, T., Eds.; Elsevier: Burlington, MA, USA, 2006; pp. 691-704.

12. Ordway, J.M.; Detloff, P.J. In vitro synthesis and cloning of long CAG repeats. Biotechniques 1996, 21, 609-610, 612.

13. Philips, A.V.; Timchenko, L.T.; Cooper, T.A. Disruption of splicing regulated by a CUG-binding protein in myotonic dystrophy. Science 1998, 280, 737-741.

14. Pujana, M.A.; Volpini, V.; Estivill, X. Large CAG/CTG repeat templates produced by PCR, usefulness for the DIRECT method of cloning genes with CAG/CTG repeat expansions. Nucleic Acids Res. 1998, 26, 1352-1353.

15. Takahashi, N.; Sasagawa, N.; Suzuki, K.; Ishiura, S. Synthesis of long trinucleotide repeats in vitro. Neurosci. Lett. 1999, 262, 45-48.

16. Hsu, R.J.; Hsiao, K.M.; Lin, M.J.; Li, C.Y.; Wang, L.C.; Chen, L.K.; Pan, H. Long tract of untranslated CAG repeats is deleterious in transgenic mice. PLoS ONE 2011, 6, e16417.

17. Peters, M.F.; Ross, C.A. Preparation of human cDNAs encoding expanded polyglutamine repeats. Neurosci. Lett. 1999, 275, 129-132.

18. Scior, A.; Preissler, S.; Koch, M.; Deuerling, E. Directed PCR-free engineering of highly repetitive DNA sequences. BMC Biotechnol. 2011, 11, 87.

19. Michalik, A.; Kazantsev, A.; van Broeckhoven, C. Method to introduce stable, expanded, polyglutamine-encoding $\mathrm{CAG} / \mathrm{CAA}$ trinucleotide repeats into $\mathrm{CAG}$ repeat-containing genes. Biotechniques 2001, 31, 250-252, 254.

20. Nakamura, Y.; Gojobori, T.; Ikemura, T. Codon usage tabulated from international DNA sequence databases: Status for the year 2000. Nucleic Acids Res. 2000, 28, 292.

21. Imbert, G.; Saudou, F.; Yvert, G.; Devys, D.; Trottier, Y.; Garnier, J.M.; Weber, C.; Mandel, J.L.; Cancel, G.; Abbas, N.; et al. Cloning of the gene for spinocerebellar ataxia 2 reveals a locus with high sensitivity to expanded CAG/glutamine repeats. Nat. Genet. 1996, 14, 285-291. 
22. Pulst, S.M.; Nechiporuk, A.; Nechiporuk, T.; Gispert, S.; Chen, X.N.; Lopes-Cendes, I.; Pearlman, S.; Starkman, S.; Orozco-Diaz, G.; Lunkes, A.; et al. Moderate expansion of a normally biallelic trinucleotide repeat in spinocerebellar ataxia type 2. Nat. Genet. 1996, 14, 269-276.

23. Sanpei, K.; Takano, H.; Igarashi, S.; Sato, T.; Oyake, M.; Sasaki, H.; Wakisaka, A.; Tashiro, K.; Ishida, Y.; Ikeuchi, T.; et al. Identification of the spinocerebellar ataxia type 2 gene using a direct identification of repeat expansion and cloning technique, DIRECT. Nat. Genet. 1996, 14, 277-284.

24. Cancel, G.; Dürr, A.; Didierjean, O.; Imbert, G.; Bürk, K.; Lezin, A.; Belal, S.; Benomar, A.; Abada-Bendib, M.; Vial, C.; et al. Molecular and clinical correlations in spinocerebellar ataxia 2: A study of 32 families. Hum. Mol. Genet. 1997, 6, 709-715.

25. Giunti, P.; Sabbadini, G.; Sweeney, M.G.; Davis, M.B.; Veneziano, L.; Mantuano, E.; Federico, A.; Plasmati, R.; Frontali, M.; Wood, N.W. The role of the SCA2 trinucleotide repeat expansion in 89 autosomal dominant cerebellar ataxia families. Frequency, clinical and genetic correlates. Brain 1998, 121 Pt 3, 459-467.

26. Mizushima, K.; Watanabe, M.; Kondo, I.; Okamoto, K.; Shizuka, M.; Abe, K.; Aoki, M.; Shoji, M. Analysis of spinocerebellar ataxia type 2 gene and haplotype analysis: (CCG) $)_{1-2}$ polymorphism and contribution to founder effect. J. Med. Genet. 1999, 36, 112-114.

27. Pujana, M.A.; Corral, J.; Gratacòs, M.; Combarros, O.; Berciano, J.; Genís, D.; Banchs, I.; Estivill, X.; Volpini, V. Spinocerebellar ataxias in spanish patients: Genetic analysis of familial and sporadic cases. The ataxia study group. Hum. Genet. 1999, 104, 516-522.

28. Choudhry, S.; Mukerji, M.; Srivastava, A.K.; Jain, S.; Brahmachari, S.K. CAG repeat instability at SCA2 locus: Anchoring CAA interruptions and linked single nucleotide polymorphisms. Hum. Mol. Genet. 2001, 10, 2437-2446.

29. Sobczak, K.; Krzyzosiak, W.J. CAG repeats containing CAA interruptions form branched hairpin structures in spinocerebellar ataxia type 2 transcripts. J. Biol. Chem. 2005, 280, 3898-3910.

30. Sobczak, K.; Michlewski, G.; de Mezer, M.; Kierzek, E.; Krol, J.; Olejniczak, M.; Kierzek, R.; Krzyzosiak, W.J. Structural diversity of triplet repeat RNAs. J. Biol. Chem. 2010, 285, 12755-12764.

31. Switonski, P.M.; Fiszer, A.; Kazmierska, K.; Kurpisz, M.; Krzyzosiak, W.J.; Figiel, M. Mouse ataxin-3 functional knock-out model. Neuromol. Med. 2011, 13, 54-65.

32. Zuker, M. Mfold web server for nucleic acid folding and hybridization prediction. Nucleic Acids Res. 2003, 31, 3406-3415.

33. Zuker, M.; Jacobson, A.B. Using reliability information to annotate RNA secondary structures. RNA 1998, 4, 669-679.

34. Waugh, A.; Gendron, P.; Altman, R.; Brown, J.W.; Case, D.; Gautheret, D.; Harvey, S.C.; Leontis, N.; Westbrook, J.; Westhof, E.; et al. RNAML: A standard syntax for exchanging RNA information. RNA 2002, 8, 707-717.

(C) 2015 by the authors; licensee MDPI, Basel, Switzerland. This article is an open access article distributed under the terms and conditions of the Creative Commons Attribution license (http://creativecommons.org/licenses/by/4.0/). 